\title{
As Atribuições de Causalidade e as Estratégias Autoprejudiciais de Alunos do Curso de Pedagogia
}

\author{
Danielle Ribeiro Ganda - Universidade Estadual de Campinas, Campinas, Brasil \\ Evely Boruchovitch - Universidade Estadual de Campinas, Campinas, Brasil
}

\begin{abstract}
Resumo
A autorregulação da aprendizagem é o processo pelo qual um aluno monitora e orienta o próprio aprendizado. Assim, por sua grande importância para uma aprendizagem de qualidade, o presente estudo teve o objetivo de investigar duas variáveis relacionadas à autorregulação: as atribuições de causalidade e o uso de estratégias autoprejudiciais. A amostra foi composta por 164 estudantes, de ambos os sexos, de 18 e 48 anos de idade, matriculados no $2^{\circ}$ e $4^{\circ}$ anos do curso de Pedagogia de universidades públicas brasileiras. A coleta de dados foi realizada por meio de duas escalas de autorrelato do tipo Likert. Os resultados indicaram que os alunos que atribuíram causas internas ao fracasso e causas externas ao sucesso foram aqueles que relataram maior frequência no uso de estratégias autoprejudiciais. Discute-se a necessidade de analisar as crenças atribucionais e o comportamento estratégico dos alunos universitários em situações de aprendizagem.

Palavras-chave: autorregulação da aprendizagem, estudantes universitários, atribuições causais, comportamento autoprejudicial
\end{abstract}

\section{The Causal Attributions and Self-Handicapping Strategies of Pedagogy Students}

\begin{abstract}
Self-regulation of learning is the process by which a student monitors and guides his own learning. Therefore, taking into account its great importance for the quality of learning, the present study aims to investigating two variables related to self-regulation: causal attributions and use of self-handicapping strategies. The sample consisted of 164 participants, of both sexes, with age range varying from 18 to 48 years old, enrolled in the second and fourth year of Pedagogy at Brazilian public universities. Data collection was carried out by means of two self-reported Likert scales. Results indicated that students who attribute failure to internal causes and success to external ones were those who reported using self-handicapping strategies more frequently. Data is discussed in terms of the need of analyzing the college student's attributional beliefs and their strategic behavior in learning situations.

Keywords: self-regulation of learning, college students, causal attributions, behavioral self-handicaps
\end{abstract}

Las Atribuciones de Causalidad y las Estrategias Auto-Perjudiciales de Alumnos del Curso de Pedagogía

\begin{abstract}
Resumen
La auto-regulación del aprendizaje es el proceso por el cual un estudiante monitorea y orienta su propio aprendizaje. Debido a su importancia para un aprendizaje de calidad, este estudio tuvo como objetivo investigar dos variables relacionadas a la auto-regulación: las atribuciones de causalidad y el uso de estrategias auto-perjudiciales. La muestra fue compuesta por 164 estudiantes, de ambos sexos, de 18 a 48 años de edad, matriculados en el $2^{\circ}$ y el $4^{\circ}$ año del curso de Pedagogía en universidades públicas de Brasil. La recolección de datos se realizó a través de dos escalas de auto-informe de tipo Likert. Los resultados indicaron que los estudiantes que atribuyeron causas internas al fracaso y causas externas al éxito fueron los que relataron el uso más frecuente de estrategias auto-perjudiciales. Se discute la necesidad de analizar las creencias de atribución y el comportamiento estratégico de estudiantes universitarios en situaciones de aprendizaje.

Palabras clave: auto-regulación del aprendizaje, estudiantes universitarios, atribuciones causales, comportamiento auto-perjudicial
\end{abstract}

Nas últimas décadas, observa-se uma rápida e profunda transformação no contexto educacional. Com o advento da tecnologia e com a expansão da Internet, as pessoas, atualmente, têm acesso a uma variedade e a uma quantidade incontável de informações e de conhecimentos que são atualizados a cada minuto. Esse fenômeno traz grandes contribuições aos processos de ensino e aprendizagem, mas, ao mesmo tempo, demanda um novo perfil de aluno e de professor. A crescente competição e especialização no mercado de trabalho requere alunos mais proativos e autônomos, bem como professores mais dinâmicos e competentes tecnicamente. Em virtude dessas novas exigências, pesquisadores têm defendido que a meta da educação, nos dias de hoje, deve ser desenvolver a autorregulação dos alunos para a aprendizagem (Bembenutty, 2011; Boruchovitch \& Ganda, 2013; Schunk, 2008).

De modo geral, a autorregulação refere-se à conduta controlada de uma pessoa, com o intuito de alcançar determinado objetivo (Zimmerman \& Schunk, 2008). Na área educacional, denomina-se de autorregulação da aprendizagem o processo pelo qual um 
aluno seleciona, organiza e monitora o seu próprio aprendizado. Trata-se de um ciclo de ações dinâmico, interdependente e particular do indivíduo que pode, didaticamente, ser dividido em três fases: prévia, do desempenho e da autorreflexão (Cho \& Bergin, 2009; Schunk, 2008; Zimmerman, 2000).

A fase prévia do ciclo da autorregulação é a que antecede a situação de aprendizagem e abrange a análise da tarefa e as crenças automotivacionais. A análise da tarefa se refere ao estabelecimento de metas e ao planejamento estratégico que nortearão a realização da atividade. As crenças automotivacionais englobam as percepções pessoais de capacidade do aluno como a autoeficácia, as expectativas de resultado e a motivação/interesse intrínseco frente à tarefa acadêmica que lhe é proposta (Schunk, 2008; Zimmerman, 2000).

A fase do desempenho diz respeito à conduta do indivíduo durante a execução da tarefa. Nessa etapa, são acionados os mecanismos de autocontrole e de automonitoramento que envolvem, por exemplo, o uso de estratégias que favorecem a aprendizagem, o controle da concentração durante o estudo e a avaliação do desempenho ao longo da atividade. $\mathrm{O}$ aluno realiza a atividade a partir do planejamento elaborado na fase anterior e monitora constantemente os seus comportamentos e pensamentos de modo a maximizar sua aprendizagem (Cho \& Bergin, 2009; Zimmerman, 2000).

Por fim, a terceira fase, posterior à realização da tarefa, é a da autorreflexão, na qual o aluno faz um autojulgamento do seu desempenho e se prepara para uma próxima situação de aprendizagem. Nesse momento, o indivíduo avalia a sua ação, atribui os resultados obtidos a alguma causa e demonstra reações emocionais diante deles. Casos, por exemplo, nos quais o estudante é bem-sucedido em uma atividade, podem levar a uma análise positiva das estratégias que usou para realizá-la e sentimentos de satisfação e orgulho por ter conseguido cumpri-la. Entretanto, situações nas quais o resultado acadêmico é insatisfatório podem gerar no aluno sentimentos de incapacidade e de frustração, causando um abalo em sua autoestima e, muitas vezes, levando-o a apresentar comportamentos prejudiciais à sua aprendizagem (Schunk, 2008; Zimmerman, 2000).

Observa-se, portanto, o impacto que a terceira etapa do ciclo exerce sobre as crenças, as emoções e o comportamento dos alunos frente à realização de atividades futuras (Zimmerman \& Schunk, 2008). Diante da importância desses fatores para a aprendizagem, buscou-se, no presente estudo, investigar duas variáveis presentes nessa última fase do processo de autorregulação: a atribuição de causalidade e as estratégias autoprejudiciais, que serão descritas sucintamente a seguir.

A atribuição de causalidade se refere ao ato de as pessoas interpretarem, ou seja, atribuírem causas aos eventos que ocorrem com elas e com os outros (Martini \& Del Prette, 2009; Weiner, 2004; 2010). De modo geral, pode-se dizer que as atribuições causais são crenças pessoais que influenciam o desempenho, a afetividade, as expectativas de sucesso e fracasso acadêmico, o comportamento em sala de aula e a motivação para aprender dos estudantes (Figueira \& Lobo, 2012; Martini \& Del Prette, 2005; Weiner, 2010). Na área educacional, as atribuições são feitas para situações de sucesso e de fracasso referentes à aprendizagem, como tirar uma boa nota na prova ou obter a reprovação em uma disciplina. A literatura indica que as seis principais causas mais mencionadas por alunos e professores diante do bom e do mau rendimento no contexto escolar são: o esforço, a capacidade, a dificuldade da tarefa, a sorte, o uso de estratégias de aprendizagem e o papel do professor (Weiner, 2004).

As causas, segundo Weiner (2004), podem ser classificadas quanto à sua localização/internalidade, estabilidade e controlabilidade. A localização ou internalidade se refere ao fato de a causa ser interna ou externa ao indivíduo. Por exemplo, capacidade e esforço poderiam ser classificados como intrínsecos às pessoas, ao passo que o auxílio do professor e a dificuldade da tarefa seriam fatores externos. Com relação à estabilidade, as causas que se mantêm ao longo do tempo são definidas como estáveis e as que se alteram, como instáveis. Dessa forma, capacidade poderia ser considerada estável e o uso de estratégias de aprendizagem como instável. No que concerne à controlabilidade, a causa pode ser caracterizada pelo aluno como controlável (ex.: aplicação de esforço) ou incontrolável (ex.: sorte, dificuldade da tarefa).

Um fator importante a ser ressaltado é que a classificação das causas é subjetiva, uma vez que é feita conforme as percepções que as pessoas têm de si mesmas e dos outros (Ganda \& Boruchovitch, 2011; Martini \& Del Prette, 2005; Weiner, 2008). Um aluno pode acreditar, por exemplo, que capacidade seja algo instável, que pode ser aprimorada com a aprendizagem, ao passo que outro estudante pode pensar que ser ou não capaz é algo predeterminado pela genética, que ele não pode fazer nada para mudar. Essas duas percepções distintas têm importantes implicações para a aprendizagem, pois conduzirão esses alunos 
a comportamentos e sentimentos diferentes frente ao sucesso e ao fracasso que vivenciarem durante a sua escolarização. Mais precisamente, casos em que o aluno passa a acreditar que o seu mau desempenho está sempre associado a fatores internos e estáveis, como a falta de capacidade, podem gerar uma queda no interesse pelos estudos e na dedicação às atividades escolares, podendo, inclusive, levá-lo a adotar comportamentos desfavoráveis à sua aprendizagem, como as estratégias autoprejudiciais (Finez \& Sherman, 2012; Woolfson, Grant, \& Campbell, 2007).

As estratégias autoprejudiciais, por sua vez, são atos ou escolhas feitos anteriormente ou durante uma atividade e que prejudicam a sua realização (Berglas \& Jones, 1978; Gadbois \& Sturgeon, 2011; Woolfson et al., 2007). Em geral, o uso de estratégias autoprejudiciais ocorre em situações em que o indivíduo não se sente plenamente capaz para realizar o que lhe é proposto. Assim, como tem a expectativa de que irá fracassar, ele cria obstáculos à boa execução da tarefa, de modo que, se de fato falhar, o fracasso seja associado aos impedimentos e não à sua falta de capacidade (Finez \& Sherman, 2012). Percebe-se, desse modo, que há uma preocupação da pessoa quanto ao julgamento dos outros frente ao seu desempenho, motivo pelo qual as pesquisas evidenciam que os indivíduos com baixa autoestima apresentam maior incidência no uso de estratégias autoprejudiciais.

No contexto acadêmico, os alunos universitários podem apresentar estratégias autoprejudiciais, tais como: abusar de álcool e de drogas, procrastinar, sair na noite anterior a um exame, fazer trabalhos na última hora, relatar sintomas físicos e psicológicos (dores de cabeça, mal-estar, nervosismo), entre outros (Ganda \& Boruchovitch, 2015; Zanatto, 2007). Algumas das consequências do uso frequente desses comportamentos são o aumento da ansiedade e do estresse, a queda da motivação e do rendimento acadêmico, além do fortalecimento de crenças negativas do aluno sobre sua própria capacidade em aprender.

De acordo com a literatura, alunos autorregulados apresentam atribuições mais adaptativas à aprendizagem e menor frequência no uso de estratégias autoprejudiciais (Bertrams \& Dickhauser, 2012; Zimmerman, 2011). Educadores ressaltam a importância de que os estudantes universitários, sobretudo os de curso de formação de professores, aprendam e aprimorem a autorregulação da sua própria aprendizagem, para que estejam mais aptos a desenvolver esse mesmo processo nos seus futuros alunos (Boruchovitch \&
Ganda, 2013; Bembenutty, 2011). Nesse sentido, a presente pesquisa buscou investigar as atribuições causais e o uso de estratégias autoprejudiciais por estudantes de um curso de Pedagogia e as possíveis relações entre essas duas variáveis, essenciais para a promoção da aprendizagem autorregulada.

\section{Método}

\section{Participantes}

A amostra, selecionada por conveniência, foi composta por 164 estudantes do curso de Pedagogia de universidades públicas brasileiras, sendo 147 (89,63\%) do sexo feminino e 17 (10,37\%) do sexo masculino. A idade dos participantes variou entre 18 e 48 anos, com média de 23,76 anos, divididos nas seguintes faixas etárias: 53 (32,32\%) entre 18 e 20 anos, 97 (59,15\%) entre 21 e 30 anos e 14 (8,54\%) entre 31 e 48 anos. Quanto ao ano de curso, $37,2 \%(n=61)$ estavam matriculados no segundo ano e $62,8 \%(n=103)$ no quarto ano.

\section{Procedimento de Coleta de Dados}

Inicialmente, foram agendadas reuniões com os coordenadores dos cursos de Pedagogia de duas universidades públicas brasileiras, nas quais thes foram entregues cartas de apresentação requerendo consentimento para a realização da pesquisa. Com a anuência dos coordenadores, o projeto foi submetido e aprovado pelo Comitê de Ética em Pesquisa da Comitê de Ética em Pesquisa da Faculdade de Ciências Médicas da UNICAMP (Protocolo no 1195/2009), em concordância com a Resolução 466/12 do Conselho Nacional de Saúde, que regulamenta os cuidados éticos a serem tomados em pesquisas realizadas com seres humanos.

Após essa etapa, foram realizados dois estudos-piloto com o intuito de aperfeiçoar os instrumentos da pesquisa. A partir desses estudos, foram feitas modificações nos enunciados das escalas e incluídos novos itens na escala de estratégias autoprejudiciais. Em seguida, a pesquisa foi apresentada aos alunos de Pedagogia das instituições contatadas e, aqueles que concordaram em participar, preencheram, em duas vias, um Termo de Consentimento Livre e Esclarecido, que garantia ser a sua participação voluntária e que os dados de identificação, tanto dos estudantes quanto das universidades, seriam mantidos em total sigilo. A coleta foi realizada coletivamente, em sala de aula, em dias e horários pré-agendados e de melhor conveniência para os alunos e para as instituições, com duração de, aproximadamente, 50 minutos. 


\section{Instrumentos}

Escala de Avaliação das Atribuições de Causalidade para Sucesso e Fracasso Acadêmico de universitários (Boruchovitch \& Santos, 2009). A escala foi formada, inicialmente, por 55 itens relativos às atribuições causais em situações de sucesso e fracasso acadêmico, com quatro opções de resposta distribuídas em formato Likert, variando de 1 (Não tem nada a ver comigo) a 4 (Me descreve realmente bem). Após a análise dos índices de consistência interna da escala, excluíram-se os itens que não funcionaram bem, resultando em 39 itens. A pontuação total da escala pode variar entre 39 e 156 pontos. Quanto maior o escore, maior a tendência de a pessoa fazer atribuições causais a fatores internos e controláveis em situações do contexto educacional. A consistência interna da escala total, aferida pelo alfa de Cronbach, foi de 0,79, índice considerado satisfatório (Prietto \& Muñiz, 2000).

A escala foi subdividida em quatro subescalas, classificadas de acordo com os exemplos de sucesso e fracasso acadêmico e as dimensões atribucionais de internalidade/lócus e controlabilidade. As subescalas são: a) internalidade frente ao sucesso acadêmico (ex.: "Tiro boas notas porque sou inteligente."), b) internalidade frente ao fracasso acadêmico (ex.: "Vou mal porque não sou esforçado.”), c) controlabilidade frente ao sucesso acadêmico (ex.: "Consigo melhorar minhas notas quando preciso.") e d) controlabilidade frente ao fracasso acadêmico (ex.: "Não consigo fazer nada para que as avaliações sejam mais fáceis."). As subescalas contêm, respectivamente, 10; 7; 12 e 10 itens e possuem as seguintes consistências internas: 0,$75 ; 0,60 ; 0,84$ e 0,81 . Na escala, há 12 itens com pontuação invertida, como a) item 06: Tiro boas notas porque tenho bons professores e b) item 34: Não consigo fazer nada para ser mais inteligente.

Escala de Estratégias Autoprejudiciais (Boruchovitch \& Ganda, 2009). A escala foi composta, a princípio, por 24 itens relativos ao uso de estratégias em contexto acadêmico, com quatro opções de resposta em formato Likert, variando entre 1 (Não tem nada a ver comigo) e 4 (Me descreve realmente bem). Assim como a escala de atribuição, também se realizou a análise dos índices de consistência interna do instrumento de estratégias autoprejudiciais, que resultou na exclusão de três itens, ficando ao todo 21. A pontuação total da escala pode alcançar o mínimo de 21 pontos e o máximo de 84 pontos, de tal modo que, quanto maior o valor, mais frequente é o relato de uso das estratégias autoprejudiciais pelos alunos. A consistência interna da escala total, aferida pelo alfa de Cronbach, foi alta, tendo-se obtido o valor de 0,84 (Prietto \& Muñiz, 2000).

Inicialmente, os itens da escala foram agrupados de acordo com seu conteúdo, em três subescalas: 1) problemas no gerenciamento do tempo (ex.: "Alguns alunos deixam para estudar nas vésperas da prova. Caso se saiam mal, dizem que não tiveram tempo suficiente para estudar toda a matéria."), 2) falta de controle da atenção (ex.: "Alguns alunos ficam mexendo no celular durante as aulas. Caso não tenham um bom resultado, dizem que foi porque não entenderam a explicação do professor.") e 3) problemas na preparação de uma atividade (ex.: "Alguns alunos não se preparam bem para uma apresentação oral e então ficam muito nervosos na hora da exposição. Caso não tenham um bom desempenho, dizem que o nervosismo os atrapalhou."). As subescalas contiveram, respectivamente, 12,5 e 4 itens e possuem as seguintes consistências internas: 0,$80 ; 0,63$ e 0,61 , todas consideradas aceitáveis (Prietto \& Muñiz, 2000). Não há itens a serem invertidos nessa escala.

\section{Procedimento de Análise de Dados}

Os dados foram analisados de acordo com os procedimentos da estatística descritiva e inferencial não paramétrica, visto que o teste de Shapiro-Wilk revelou a ausência da distribuição normal dos dados. Foram computados os cálculos dos valores totais, médias e desvios-padrão e estimada a correlação entre as duas variáveis por meio do coeficiente de Spearman, adotando um nível de significância de $5 \%$.

\section{Resultados}

$\mathrm{Na}$ Tabela 1, encontram-se descritos os dados da escala de atribuição de causalidade. As médias e os desvios-padrão das subescalas foram: a) internalidade/ sucesso $(M=27,54$ e $D P=4,77)$, b) internalidade/fracasso $(M=12,94$ e $D P=2,77)$, c) controlabilidade/ sucesso $(M=34,72$ e $D P=7,33)$ e d) controlabilidade/ fracasso $(M=36,62$ e $D P=4,23)$. O valor total mínimo alcançado pela amostra na escala completa foi 67 e o máximo 137, com desvio-padrão de 11,21. Esses resultados revelam que um número expressivo de alunos relata que as causas responsáveis pelo seu sucesso e fracasso na universidade são internas e controláveis.

Como mostra a Tabela 2, as médias e os desvios-padrão das subescalas de estratégias autoprejudiciais foram: a) problemas no gerenciamento do tempo ( $M$ $=20,24$ e $D P=5,61)$, b) falta de controle da atenção 
$(M=6,79$ e $D P=2,10)$ e c) problemas na preparação de uma atividade $(M=7,08$ e $D P=2,22)$. Na escala completa, o valor mínimo alcançado pela amostra foi 21, e o máximo 54, com desvio-padrão de 8,39. Esses dados revelam que, de modo geral, os alunos relataram de pouco a moderado o uso de estratégias autoprejudiciais em contexto acadêmico. Foi interessante notar, também, que alguns afirmaram não usar nenhuma das mencionadas nos itens da escala. As correlações entre os escores totais das escalas e subescalas de atribuição de causalidade e de estratégias autoprejudiciais também foram estimadas (Tabela 3).

Os resultados evidenciam que os escores na subescala Problemas no gerenciamento do tempo se

Tabela 1

Média, Desvio-Padrão e Escores Minimo e Máximo na Escala e Subescalas de Atribuição de Causalidade

\begin{tabular}{lccccc}
\hline & $\mathrm{N}$ & Média & D.P. & Mínimo & Máximo \\
\hline Internalidade frente ao sucesso acadêmico & 164 & 27,54 & 4,77 & 14 & 40 \\
Internalidade frente ao fracasso acadêmico & 163 & 12,94 & 2,77 & 8 & 26 \\
Controlabilidade frente ao sucesso acadêmico & 160 & 34,72 & 7,33 & 15 & 48 \\
Controlabilidade frente ao fracasso acadêmico & 162 & 36,62 & 4,23 & 13 & 40 \\
Atribuição de causalidade total & 160 & 111,78 & 11,21 & 67 & 137 \\
\hline
\end{tabular}

Tabela 2

Média, Desvio-Padrão e Escores Mínimo e Máximo na Escala e Subescalas de Estratégias Autoprejudiciais

\begin{tabular}{lccccc}
\hline & $\mathrm{N}$ & Média & DP & Mínimo & Máximo \\
\hline Problemas no gerenciamento do tempo & 164 & 20,24 & 5,61 & 12 & 35 \\
Falta de controle da atenção & 164 & 6,79 & 2,10 & 5 & 17 \\
Problemas na preparação de uma atividade & 164 & 7,08 & 2,22 & 4 & 15 \\
Estratégias autoprejudiciais total & 164 & 34,12 & 8,39 & 21 & 54 \\
\hline
\end{tabular}

Tabela 3

Correlaçẽes dos Escores das Escalas e Subescalas de Atribuição de Causalidade e de Estratégias Autoprejudiciais

\begin{tabular}{|c|c|c|c|c|c|c|}
\hline & & $\begin{array}{l}\text { Internalidade/ } \\
\text { sucesso }\end{array}$ & $\begin{array}{l}\text { Internalidade/ } \\
\text { fracasso }\end{array}$ & $\begin{array}{l}\text { Controlabilidade/ } \\
\text { sucesso }\end{array}$ & $\begin{array}{l}\text { Controlabilidade/ } \\
\text { fracasso }\end{array}$ & $\begin{array}{l}\text { Atribuição de } \\
\text { causalidade total }\end{array}$ \\
\hline $\begin{array}{l}\text { Problemas no } \\
\text { gerenciamento } \\
\text { do tempo }\end{array}$ & $\begin{array}{l}r= \\
p=\end{array}$ & $\begin{array}{l}-0,31 \\
<, 0001\end{array}$ & $\begin{array}{l}0,47 \\
<, 0001\end{array}$ & $\begin{array}{l}0,07 \\
0,354\end{array}$ & $\begin{array}{l}-0,30 \\
<, 0001\end{array}$ & $\begin{array}{l}-0,76 \\
0,338\end{array}$ \\
\hline $\begin{array}{l}\text { Falta de controle } \\
\text { da atenção }\end{array}$ & $\begin{array}{l}r= \\
p=\end{array}$ & $\begin{array}{l}-0,28 \\
<, 0001\end{array}$ & $\begin{array}{l}0,33 \\
<, 0001\end{array}$ & $\begin{array}{l}-0,03 \\
0,969\end{array}$ & $\begin{array}{l}-0,12 \\
0,127\end{array}$ & $\begin{array}{l}-0,97 \\
0,221\end{array}$ \\
\hline $\begin{array}{l}\text { Problemas na } \\
\text { preparação de } \\
\text { uma atividade }\end{array}$ & $\begin{array}{l}r= \\
p=\end{array}$ & $\begin{array}{l}-0,35 \\
<, 0001\end{array}$ & $\begin{array}{l}0,38 \\
<, 0001\end{array}$ & $\begin{array}{l}-0,06 \\
0,418\end{array}$ & $\begin{array}{l}-0,17 \\
0,028\end{array}$ & $\begin{array}{l}-0,17 \\
0,030\end{array}$ \\
\hline $\begin{array}{l}\text { Estratégias } \\
\text { autoprejudiciais } \\
\text { total }\end{array}$ & $\begin{array}{l}r= \\
p=\end{array}$ & $\begin{array}{l}-0,37 \\
<, 0001\end{array}$ & $\begin{array}{l}0,51 \\
<, 0001\end{array}$ & $\begin{array}{l}0,04 \\
0.597\end{array}$ & $\begin{array}{l}-0,29 \\
<, 0001\end{array}$ & $\begin{array}{l}-0,11 \\
0,143\end{array}$ \\
\hline
\end{tabular}

Nota. $* r=$ coeficiente de correlação de Spearman; $p=$ valor-p; 
correlacionam de forma significante, positiva e moderada com a Internalidade/fracasso e significante, negativa e moderada com a Internalidade/ sucesso e a Controlabilidade/ fracasso. Observa-se, desse modo, que os alunos com mais problemas na organização do tempo dedicado ao estudo tendem a atribuir causas internas e incontroláveis às situações de fracasso e causas externas ao sucesso.

Houve, também, correlações significantes entre a subescala de estratégias autoprejudiciais relacionadas à "falta de controle da atenção" e as subescalas de atribuição: Internalidade/ fracasso (correlação positiva fraca) e Internalidade/sucesso (correlação negativa moderada). Portanto, pode-se inferir que os universitários que relataram ter mais problemas no controle de sua atenção tendem a internalizar mais o fracasso e externalizar mais o sucesso na realização das atividades acadêmicas.

Os escores na escala de estratégias autoprejudiciais total se correlacionam moderada e negativamente com as subescalas Internalidade/sucesso, Controlabilidade/ fracasso, mas positivamente com a Internalidade/fracasso. Os dados relevam que os alunos que usam essas estratégias com mais frequência acreditam que o fracasso ocorra por fatores internos e incontroláveis e consideram que o sucesso seja desencadeado predominantemente por fatores externos a si próprios.

Enfim, constatou-se que os escores na subescala Problemas na preparação se correlacionaram de modo positivo e moderado com a subescala Internalidade/ fracasso, negativo e moderado com a Internalidade/ sucesso e negativo e fraco com a subescala Controlabilidade/fracasso assim como com o total da escala de atribuição de causalidade. Em conjunto, esses resultados sugerem que os universitários que usam mais estratégias prejudiciais ao se prepararem para a realização de uma atividade acadêmica, acreditam que o fracasso advenha de fatores internos e incontroláveis. Entretanto, os alunos que reportaram uso mais frequente desse tipo de estratégia tendem a se responsabilizar pelos resultados alcançados na universidade, relatando que são desencadeados por causas internas e controláveis.

\section{Discussão}

O presente estudo teve como objetivo analisar as atribuições de causalidade e o uso de estratégias autoprejudiciais de alunos universitários e as possíveis correlações entre essas duas variáveis. Inicialmente, observou-se, pela análise dos dados da escala de atribuição de causalidade, que um número expressivo de alunos acredita que as causas responsáveis pelo seu desempenho sejam internas e controláveis, resultado este que está em consonância com outras pesquisas (Cortés-Suarez \& Sandiford, 2008; Ferla, Valcke, \& Schuyten, 2009; Haugen, Lund, \& Ommundsen, 2008).

Em linhas gerais, os estudos revelam que mesmo alunos provenientes de diferentes culturas, cursos e níveis de rendimento acadêmico relatam que o sucesso e o fracasso na faculdade são decorrentes, principalmente, da aplicação (ou não) de esforço e de dedicação, que são fatores internos e controláveis pelo indivíduo (Ferla et al., 2009; Haugen et al., 2008). Tendo em vista o processo de autorregulação da aprendizagem, esses resultados são muito relevantes, pois alunos autorregulados tendem a possuir atribuições favoráveis à aprendizagem, ou seja, eles acreditam que o sucesso dependa predominantemente de seu empenho e do comprometimento com os estudos (Bertrams \& Dickhauser, 2012; Sitzman \& Ely, 2010; Zimmerman \& Schunk, 2008; Woolfson, et al., 2007).

No que tange à escala de estratégias autoprejudiciais, constatou-se pouco relato de uso dessas estratégias entre os participantes. Esse resultado é igualmente animador, se for consideradoo impacto negativo do uso das estratégias autoprejudiciais para a aprendizagem. Contudo, embora pouco frequente, observou-se que parte dos universitários revelou que eventualmente as utiliza em situações acadêmicas, tal como se observa em outras pesquisas (Gadbois \& Sturgeon, 2011; Martin, Marsh, \& Debus, 2001; Schraw, Wadkins, \& Olafson, 2007). Desse modo, recomenda-se que futuros estudos investiguem, especificadamente, quais são as estratégias mais utilizadas pelos alunos, em quais tipos de atividades acadêmicas são mais aplicadas e por quê. Sugere-se também que sejam desenvolvidas e testadas propostas pedagógicas voltadas a auxiliar os estudantes a substituir o uso de estratégias autoprejudiciais pelo uso de estratégias favoráveis à aprendizagem dentro do contexto acadêmico.

A análise da relação entre os escores totais das escalas e subescalas revelou dados também interessantes. Os participantes que relataram uso mais frequente de estratégias autoprejudiciais afirmaram que o fracasso é causado por fatores internos e incontroláveis, ao passo que o sucesso é decorrente de causas externas. Esses achados divergem em parte da literatura segundo a qual alunos mais autorregulados empregam menos estratégias autoprejudiciais e apresentam atribuições mais adequadas à aprendizagem (internas e controláveis) tanto em situações de êxito como de fracasso 
acadêmico (Alter \& Forgas, 2007; Zimmerman \& Schunk, 2004). Entretanto, o resultado é importante, pois pressupõe a ideia de que esses universitários tendem a internalizar o fracasso e atribuir o sucesso a fatores externos por, possivelmente, perceberem que têm hábitos prejudiciais ao estudo, como a procrastinação, a falta de leitura de textos e a distração durante as aulas, entre outros. Foi interessante notar que mesmo os alunos que mais reportaram utilizar as estratégias autoprejudiciais, tenderam a assumir a responsabilidade pelo seu mau desempenho.

No que se refere aos problemas relacionados à falha de controle da atenção e à falta de preparo, os universitários que relataram engajar-se nesses comportamentos com mais frequência, afirmaram que o sucesso é determinado por causas externas e o fracasso por motivos internos, tanto controláveis como incontroláveis. A percepção de controlabilidade afeta diretamente as emoções, o comportamento e as expectativas do aluno perante as atividades acadêmicas (Figueira \& Lobo, 2012; Martini \& Boruchovitch, 2004; Weiner, 2004). A crença de que as causas que acarretam um fracasso são incontroláveis suscita sentimentos de desesperança e de desmotivação, uma vez que não se pode fazer nada para evitar o mau desempenho (Figueira \& Lobo, 2012; Ganda \& Boruchovitch, 2011; Martini \& Del Prette, 2005; 2009). Dessa forma, pode-se supor que, para evitar ser responsabilizado pelo fracasso, o aluno tenda a recorrer mais frequentemente ao uso de estratégias autoprejudiciais, o que explicaria parte dos dados aqui encontrados.

Os participantes que relataram ter maiores problemas no gerenciamento do tempo acreditam que o fracasso acadêmico ocorra devido a causas internas e incontroláveis e que o sucesso, a fatores externos. De acordo com Finez e Scherman (2012), quando o estudante não se sente capaz em realizar uma atividade, ele pode despender mais tempo na "criação de desculpas" para o seu possível fracasso do que na própria realização da tarefa. Depois, ao ter um fraco desempenho ele tende a associá-lo a causas incontroláveis e nem sempre condizentes com a realidade, como a falta de capacidade ou a má sorte.

Em síntese, os dados encontrados na presente pesquisa foram interessantes e a maior parte deles muito favorável, ao revelar que estudantes que aspiram a ser professores reportam engajamento em comportamentos promotores de um bom desempenho acadêmico e parecem ter atribuições compatíveis com a aprendizagem de qualidade. Todavia, cabe lembrar que as escalas empregadas na coleta de dados foram utilizadas pela primeira vez na presente pesquisa e, ainda que tenham sido pré-testadas em estudos-piloto e tenham apresentado boa consistência interna, devem ser objeto de futuras investigações, tendo em vista o seu refinamento. Recomenda-se, pois, que sejam aplicadas a uma amostra maior de estudantes universitários, inclusive de outros cursos, além da Pedagogia. Ademais, como o presente estudo teve resultados animadores e se pautou apenas em instrumentos de autorrelato, destaca-se a necessidade de que pesquisas futuras incluam também na coleta de dados, medidas de observação de comportamento.

\section{Considerações Finais}

Acredita-se que os resultados do presente estudo possam trazer importantes contribuições à pesquisa na área educacional, especialmente na que concerne aos cursos de formação de professores. As atribuições e o uso de estratégias autoprejudiciais têm forte influência na conduta e na motivação dos alunos durante a faculdade e, possivelmente, em sua vida profissional futura. Desse modo, trabalhar com universitários em curso de formação de professores é fundamental, uma vez que as pesquisas têm constatado que as crenças dos docentes afetam o seu comportamento em sala, as suas expectativas de sucesso e fracasso acadêmico e o desempenho de seus alunos (Berry, 2006; Boruchovitch \& Ganda, 2013; Warfield, Wood, \& Lehman, 2005).

Estudos realizados no contexto brasileiro revelam que o ensino de estratégias e métodos que auxiliem a aprendizagem dos estudantes é infrutífero, se os próprios docentes não acreditarem na capacidade de seus alunos em aprender e na sua capacidade em ensiná-los (Costa \& Boruchovitch, 2006; Martini \& Del Prette, 2009). Dessa forma, torna-se fundamental que cursos como os de Pedagogia e as Licenciaturas invistam não somente em conteúdos didáticos e pedagógicos, mas também no ensino e na reflexão dos alunos acerca dos demais componentes motivacionais e comportamentais presentes na aprendizagem, o que pode ser realizado por meio da promoção da autorregulação (Bembenutty, 2011; Boruchovitch \& Ganda, 2013).

Tendo em vista esse objetivo, seria importante que se fomentassem nas faculdades propostas de ensino voltadas ao desenvolvimento da aprendizagem autorregulada, tais como: cursos complementares, disciplinas específicas, atividades extras, entre outras. Essas propostas visariam dar a oportunidade ao universitário de refletir sobre a sua conduta acadêmica 
e de ser orientado sobre métodos que o ajudem a potencializar o aprendizado e melhorar o rendimento, buscando, assim, tornar-se um aluno e, até mesmo, um futuro profissional mais autorregulado (Boruchovitch \& Ganda, 2013; Costa \& Boruchovitch, 2006; Woolfson et al., 2007).

\section{Referências}

Alter, A., \& Forgas, J. (2007). On being happy but fearing failure: The effects of mood on self-handicapping strategies. Journal of Experimental Social Psychology, 43(6), 947-954. doi:10.1016/j.jesp.2006.07.009

Bembenutty, H. (2011). New directions for self-regulation of learning in postsecondary education. New Directions for Teaching and Learning, 126, 117-124. doi: 10.1002/tl.450

Berglas, S., \& Jones, E. E. (1978). Drug choice as a self-handicapping strategy in response to noncontingent success. Journal of Personality and Social Psychology, 36(4), 405-417. Recuperado de http:// www.ncbi.nlm.nih.gov/pubmed/650387

Berry, R. A. W. (2006). Beyond strategies: Teacher beliefs and writing instruction in two primary inclusion classroom. Journal of Learning Disabilities, 39(1), 11-24. Recuperado de http:// eric.ed.gov/?id=EJ757898

Bertrams, A. \& Dickhauser, O. (2012). Thinkers feel better self-control capacity as mediator of the relationship between need for cognition and affective adjustment. Journal of Individual Differences, 33(2), 69-75.

Boruchovitch, E., \& Ganda, D. R. (2009). Escala de estratégias autoprejudiciais (Manuscrito não publicado). Universidade Estadual de Campinas, São Paulo, SP.

Boruchovitch, E., \& Ganda, D. R. (2013). Fostering self-regulated skills in an educational psychology course for brazilian preservice teachers. Journal of Cognitive Educatioan and Psychology, 12(2), 157-177. doi: 10.1891/1945-8959.12.2.157

Boruchovitch, E., \& Santos, A. A. A. (2009). Escala de Avaliação das Atribuições de Causalidade para Sucesso e Fracasso Acadêmico de Universitários (Manuscrito não publicado). Universidade Estadual de Campinas, São Paulo, SP.

Cho, M. H., \& Bergin, D. A. (2009). Review of self-regulated learning models and implications for theory development.
Paper presented at American Educational Research Association Annual Meeting, San Diego, CA.

Cortés-Suárez, G., \& Sandiford, J. R. (2008). Causal attributions for success or failure of students in college algebra. Community College Journal of Research and Practice, 32, 325-346. Reuperado de http://eric. ed.gov/?id=EJ787014

Costa, E., \& Boruchovitch, E. (2006). A autoeeficácia e a motivação para aprender. Considerações para o desempenho escolar dos alunos. Em Azzi, R. G. \& Polydoro, S. A. J. (Eds.). Autoeficácia em diferentes contextos. Campinas, SP: Alínea.

Ferla, J., Valcke, M., \& Schuyten, G. (2009). Student models of learning and their impact in study strategies. Studies in Higher Education, 34(2), 185-202. Recuperado de http://eric.ed.gov/?id=EJ832833

Figueira, A. P. C., \& Lobo, R. A. C. T. (2012). A trilogia atribuições causais, concepções pessoais de inteligência e mensagens de feedback: Um contributo para a compreensão do fracasso escolar. Práxis Educacional, 8(13), 83-105. Recuperado de http:// periodicos.uesb.br/index.php/praxis/article/ view $/ 1581 / 0$

Finez, L., \& Sherman, D. K. (2012). Train in vain: The role of the self in claimed self-handicapping strategies. Journal of Sport and Exercise Psychology, 34(2), 600-620. Recuperado de http://www.humankinetics.com/acucustom/sitename/Documents / DocumentItem/03_Finez_0009.pdf

Gadbois, S. A., \& Sturgeon, R. D. (2011). Academic self-handicapping: Relationships with learning specific and general self-perceptions and academic performance over time. British Journal of Educational Psychology, 81(2), 207-222. doi: 10.1348/000709910X522186

Ganda, D. R., \& Boruchovitch, E. (2011). Atribuição de causalidade no ensino superior: Análise da produção científica. Estudos Interdisciplinares em Psicologia, 2(1), 2-18. Recuperado de http://pepsic.bvsalud. org/pdf/eip/v2n1/a02.pdf

Ganda, D. R., \& Boruchovitch, E. (2015). Self-handicapping strategies for learning of preservice teachers. Revista Estudos de Psicologia, 32(3), 417-425. doi: 10.1590/0103-166X2015000300007

Haugen, R., Lund, T., Ommundsen, Y. (2008). Personality dispositions, expectancy and context in attributional thinking. Scandinavian 
Journal of Educational Research, 52(2), 171-185. doi: 10.1080/00313830801915804

Martin, A., Marsh. H., \& Debus, R. (2001). Self-handicapping and defensive pessimism: Exploring a model of predictors and outcomes from a self-protection perspective. Journal of Educational Psychology, 93(1), 87-102. doi:10.1016/S0361-476X(02)00008-5

Martini, M. L., \& Boruchovitch, E. (2004). A teoria da atribuição de causalidade: Contribuições para a formação e atuação de educadores. Campinas: Alínea.

Martini, M. L., \& Del Prette, Z. (2005). Atribuições de causalidade e afetividade de alunos de alto e baixo desempenho acadêmico em situações de sucesso e fracasso escolar. Revista Interamericana de Psicologia, 39(3), 355-368. Recuperado de https:// dialnet.unirioja.es/servlet/articulo?codigo $=2989470$

Martini, M., \& Del Prette, Z. (2009). Crenças docentes e implicações para o processo de ensino-aprendizagem. Revista Semestral da Associação Brasileira de Psicologia Escolar e Educacional, 13, 75-85. Recuperado de http://www.scielo.br/pdf/pee/v13n1/ v13n1a09.pdf

Prieto, G., \& Muñiz, J. (2000). Um modelo para evaluar la calidad de los tests utilizados em España. Madrid, Espanha: Papeles Del Psicólogo. Recuperado de http://www.cop.es/tests/modelo.htm.

Schraw, G., Wadkins, T., \& Olafson, L. (2007). Doing the things we do: A grounded theory of academic procrastination. Journal of Educational Psychology, 99(1), 12-25. Recuperado de http:/ / eric. ed.gov/?id=EJ754544

Schunk, D. H. (2008). Atribution as motivators of self-regulated learning. Em D. H. Schunk \& B. J. Zimmerman (Eds.), Motivation and Self-regulated learning: Theory, research and applications (p. 245-266). England: MPG Books Group.

Sitzmann, T., \& Ely, K. (2010). Sometimes you need a reminder: The effects of prompting self-regulation on regulatory processes, learning, and attrition. Journal of Applied Psychology, 95(1), 132-144. Recuperado de http://business2.ucdenver.edu/ research $/$ ? $=392$

Warfield, J., Wood, T., \& Lehman, J. D. (2005). Autonomy, beliefs and the learning of elementary mathematics teachers. Teaching and Teacher Education, 21(4), 439-459. doi:10.1016/j.tate.2005.01.011
Weiner, B. (2004). Attribution theory revisited: Transforming cultural plurality into theoretical unity. Em Research on Sociocultural influences on motivation and learning. Information Age Publishing, Inc., 13-29.

Weiner, B. (2008). Reflections on the history of attribution theory and research. Social Psychology, 39(3), 151-156. doi: 10.1027/1864-9335.39.3.151

Weiner, B. (2010). The development of an attribution-based theory of motivation: A history of ideas. Educational Psychologist, 45(1), 28-36. doi: 10.1080/00461520903433596

Woolfson, L., Grant, E., \& Campbell, L. (2007). A comparison of special, general and support teachers controllability and stability attributions for children's difficulties in learning. Educational Psychology, 27(2), 295-306. Recuperado de http://eric. ed.gov/?id=EJ764153

Zanatto, R. (2007). Perfil motivacional de alunos de arquitetura: Um estudo exploratório (Dissertação de mestrado). Faculdade de Educação, Universidade Estadual de Londrina: Londrina, Paraná.

Zimmerman, B. J. (2000). Attaining self-regulation: A social cognitive perspective. Em M. Boekaerts, P. R. Pintrich \& M. Zeidner (Eds.), Handbook of selfregulation (p. 13-39). San Diego: Academic Press.

Zimmerman, B. J. (2011). Motivational sources and outcomes of self-regulated learning and performance. Em B. J. Zimmerman \& D. H. Schunk (Eds.), Handbook of self-regulation of learning and performance (pp. 49-64). New York, NY: Routledge, Taylor \& Francis Group.

Zimmerman, B. J. \& Schunk, D. H. (2004). Self-regulating intellectual processes and outcomes: a Social cognitive perspective. Em D. Y. Dai \& R. J. Sternberg (Eds.), Motivation, emotion and cognition: integrative perspectives on intellectual development and functioning ( $\mathrm{p}$. 323-350). United States: Lawrence Erlbaum Associates Inc.

Zimmerman, B. J., \& Schunk, D. H. (2008). Motivation: An essential dimension of self-regulated learning. Em D. H. Schunk \& B. J. Zimmerman (Eds.), Motivation and self-regulated learning: Theory, research and applications (p. 1-30). England: MPG Books Group.

Recebido em: 16/02/2015

Reformulado em: 23/06/2015

Aprovado em: 13/07/2015 
Nota das autoras:

O artigo é baseado na dissertação: "Atribuições de causalidade e estratégias autoprejudiciais de alunos de curso de formação de professores", defendida pela primeira autora, em abril de 2011, sob a orientação da segunda autora, na Faculdade de Educação da Universidade Estadual de Campinas.

As autoras agradecem o apoio financeiro da Coordenação de Aperfeiçoamento de Pessoal de Nível Superior (CAPES) e do Conselho Nacional de Desenvolvimento Científico e Tecnológico (CNPq).

Sobre as autoras:

Danielle Ribeiro Ganda é psicóloga, mestre e doutora em Psicologia Educacional, pela Faculdade de Educação da Universidade Estadual de Campinas, e bolsista da Fundação de Amparo à Pesquisa do Estado de São Paulo - FAPESP. E-mail: danielleganda@hotmail.com

Evely Boruchovitch é psicóloga, $\mathrm{PhD}$ em Educação pela University of Southern California, Los Angeles, professora titular da Faculdade de Educação da Universidade Estadual de Campinas e bolsista de produtividade do CNPq. E-mail: evely@unicamp.br

\section{Contato com as autoras:}

Danielle Ribeiro Ganda

Rua Tapajós, 800, apto. 201, Saraiva

CEP: 38408-414

Uberlândia-MG, Brasil 